\title{
Lipid lowering for primary prevention of stroke in older adults?
}

\author{
Not yet, but new findings justify trials in this age group
}

\author{
Graeme $\mathrm{J}$ Hankey professor of neurology ${ }^{1}$ consultant neurologist ${ }^{2}$ research affiliate and honorary \\ senior research fellow ${ }^{3}$
}

${ }^{1}$ School of Medicine and Pharmacology, University of Western Australia, Harry Perkins Institute of Medical Research, QEII Medical Centre, Nedlands, Perth, Australia 6009; ${ }^{2}$ Department of Neurology, Sir Charles Gairdner Hospital, Nedlands, Perth, Australia; ${ }^{3}$ Western Australian Neuroscience Research Institute, Nedlands, Perth, Australia

Vascular events of the heart (myocardial infarction) and brain (stroke) are the world's leading cause of death and disability. Most events occur in older people without previous symptomatic vascular disease. Although this group has a lower relative risk of vascular events than people with a previous history, they are substantially more prevalent and so account for a greater absolute number of events. ${ }^{1}$

Strategies to reduce the burden of vascular events include lipid lowering with statins. Every $1.0 \mathrm{mmol} / \mathrm{L}$ reduction in low density lipoprotein cholesterol reduces major vascular events by one fifth in populations of mean age 63 years, among whom many $(60 \%)$ have previous vascular disease. ${ }^{2}$ The absolute benefit of statins is also proportional to the absolute risk of vascular events, ${ }^{2}$ so guidelines recommend adding statins to lifestyle changes if the 10 year risk exceeds $7.5-10 \% .^{34}$ Nevertheless, guidelines do not generally recommend statins for adults aged over 75 years without vascular disease because robust evidence is limited..$^{5-7}$

In a linked paper (doi:10.1136/bmj.h2335), Alpérovitch and colleagues report that use of statins or fibrates to lower serum cholesterol was associated with a one third lower risk of stroke (hazard ratio $0.66,95 \%$ confidence interval 0.49 to 0.90 ) in a French cohort of 7484 older (mean age 74 years) adults without previous vascular disease. ${ }^{8}$ They compared use of these agents with no use, over a mean follow-up of nine years. Results were consistent for ischaemic stroke (hazard ratio $0.63,0.45$ to 0.84 ) and haemorrhagic stroke $(0.72,0.37$ to 1.42$)$ and were comparable between fibrates and statins. The authors found no overall association between use of lipid lowering drugs and coronary heart disease (hazard ratio $1.12,0.90$ to 1.40 ). ${ }^{8}$

The divergent findings for stroke (decreased) and coronary heart disease (not decreased) among users of lipid lowering drugs could be due to random error (the $95 \%$ confidence intervals around the hazard ratios for stroke and coronary heart disease were wide and included a hazard ratio of 0.90 ), systematic error (such as selection or channelling bias, survival bias, or residual confounding), and the more diverse pathogenesis of stroke compared with coronary heart disease.

This is the first observational study to describe a significant association between use of lipid lowering drugs and decreased risk of stroke. ${ }^{8}$ Previous observational studies have reported only a weak positive association between total cholesterol and stroke mortality in early middle age and no association among older people. ${ }^{9}$

More reliable data, from a meta-analysis of 76 randomised trials in 251476 people of mean age 61 years, indicate that statins significantly reduce the risk of all stroke (odds ratio $0.85,0.78$ to 0.92$),{ }^{10}$ supporting the findings of Alpérovitch and colleagues. Furthermore, statins reduced non-fatal stroke (odds ratio 0.81, 0.74 to 0.89$)$ but not fatal stroke $(0.98,0.85$ to 1.13$) .{ }^{10}$ In this meta-analysis, the proportional reduction in stroke risk associated with statins was consistent for primary prevention (odds ratio $0.80,0.69$ to 0.93 ) and secondary prevention $(0.85$, 0.80 to 0.91 ) and was broadly consistent between participants aged under 60 years $(0.88,0.77$ to 1.01$)$ and older participants $(0.87,0.81$ to 0.95$) .{ }^{10}$

Among eight placebo controlled trials of statins in 24674 participants aged 65 or over (mean age 73.0 (SD 2.9 years) without previous vascular disease, statins reduced the relative risk of stroke (0.76, 0.63 to 0.93$)$ and myocardial infarction $(0.61,0.43$ to 0.85$)$ but not all cause death $(0.94,0.86$ to 1.04$)$ or cardiovascular death $(0.91,0.69$ to 1.12$)$ after 3.5 years' mean follow-up compared with placebo. ${ }^{6}$ Most of these trials had an upper age limit of 70-75 years. The only trial that specifically tested statins in adults aged 70-82 years reported that statins reduced major vascular events by $15 \%$ (hazard ratio $0.85,0.74$ to 0.91 ) compared with placebo. ${ }^{5}$ The effect was broadly comparable among people with previous vascular disease $(17.4 \%$ statin, $21.7 \%$ placebo; hazard ratio $0.78,0.66$ to 0.93$)$ and without vascular disease $(11.4 \% v 12.1 \%$; $0.94,0.77$ to 1.15 ; 
interaction $\mathrm{P}=0.19) .{ }^{5}$ However, statins had no significant effect on stroke alone (hazard ratio $1.03,0.81$ to 1.31 ) or mortality $(0.97,0.83$ to 1.14$) .^{5}$

In view of the limited evidence for lipid lowering in the primary prevention of vascular events in people aged over 75 , and the lack of any definite benefit on mortality, no definitive indication remains for statin treatment in older adults without previous vascular disease. ${ }^{34}$

The study of Alpérovitch and colleagues will not change guidelines because of its observational design and inherent potential for systematic error. ${ }^{8}$ However, the results are sufficiently compelling to justify further research testing the hypothesis that lipid lowering may be effective in the primary prevention of stroke in older people. As populations age, the burden of vascular diseases among older adults increases. Also, new options for lowering serum cholesterol are becoming available (such as ezetimibe, cholesterol ester transfer protein inhibitors, ${ }^{11}$ and proprotein convertase subtilisin-kexin type 9 inhibitors ${ }^{12}$ ). Alpérovitch and colleagues' study reinforces the need for more robust evidence from large randomised trials evaluating lipid lowering specifically in older people without previous vascular disease.

Meanwhile, for clinicians and patients, the decision to start statins for primary prevention of vascular disease in people over 75 continues to be based on sound clinical judgment after consideration of each person's predicted vascular risk without and with statins, the predicted risk of adverse effects of statins (against a backdrop of increasing comorbidities, polypharmacy, and other safety considerations), and the patient's own priorities and preferences for treatment.

Competing interests: I have read and understood the BMJ policy on declaration of interests and declare the following interests: honorariums for serving as a consultant to Sanofi Aventis by means of a single telephone call in October 2013 discussing the potential for alirocumab (antibody to PCKS9) for the prevention of recurrent stroke, for speaking at scientific symposiums about stroke prevention in atrial fibrillation sponsored by Bayer Pharmaceuticals, and for discussing stroke prevention in atrial fibrillation in an online program for theheart.org funded by Medscape.

Provenance: Commissioned; not externally peer reviewed.

1 Rose G. Strategy of prevention: lessons from cardiovascular disease. Br Med J (Clin Res Ed) 1981;282:1847-51.

2 Cholesterol Treatment Trialists' (CTT) Collaboration. Efficacy and safety of LDL-lowering therapy among men and women: meta-analysis of individual data from 174000 participants in 27 randomised trials. Lancet 2015;385:1397-405.

3 Stone NJ, Robinson JG, Lichtenstein AH, et al. 2013 ACC/AHA guideline on the treatment of blood cholesterol to reduce atherosclerotic cardiovascular risk in adults: a report of the American College of Cardiology/American Heart Association Task Force on Practice Guidelines. Circulation 2014:129(25 suppl 2):S1-45.

4 Reiner Z, Catapano AL, De Backer G, et al. ESC/EAS guidelines for the management of dyslipidaemias: the Task Force for the Management of Dyslipidaemias of the European Society of Cardiology (ESC) and the European Atherosclerosis Society (EAS). Eur Heart J 2011;32:1769-818.

5 Shepherd J, Blauw GJ, Murphy MB, et al. Pravastatin in elderly individuals at risk of vascular disease (PROSPER): a randomised controlled trial. Lancet 2002;360:1623-30

6 Savarese G, Gotto AM Jr, Paolillo S, et al. Benefits of statins in elderly subjects without established cardiovascular disease: a meta-analysis. J Am Coll Cardiol 2013;62:2090-9.

7 Taylor F, Huffman MD, Macedo AF, et al. Statins for the primary prevention of cardiovascular disease. Cochrane Database Syst Rev 2013;1:CD004816.

8 Alpérovitch $\mathrm{A}, \mathrm{Kurth} \mathrm{T}$, Bertrand $\mathrm{M}$, et al. Primary prevention with lipid lowering drug and long term risk of vascular events in older people: population based cohort study. BMJ 2015;350:h2335.

9 Prospective Studies Collaboration, Lewington S, Whitlock G, et al. Blood cholesterol and vascular mortality by age, sex, and blood pressure: a meta-analysis of individual data from 61 prospective studies with 55,000 vascular deaths. Lancet 2007;370:1829-39.

10 De Caterina R, Scarano M, Marfisi R, et al. Cholesterol-lowering interventions and stroke: insights from a meta-analysis of randomized controlled trials. J Am Coll Cardiol 2010;55:198-211.

11 Kastelein JJ, Besseling J, Shah S, et al. Anacetrapib as lipid-modifying therapy in patients with heterozygous familial hypercholesterolaemia (REALIZE): a randomised, double-blind, placebo-controlled, phase 3 study. Lancet 2015; published online 2 March.

12 Sabatine MS, Giugliano RP, Wiviott SD, et al. Efficacy and safety of evolocumab in reducing lipids and cardiovascular events. N Engl J Med 2015;372:1500-9.

Cite this as: BMJ 2015;350:h2568

๑ BMJ Publishing Group Ltd 2015 